\title{
First Results from a STIS Survey of Close Pre-Main Sequence Binaries in Taurus
}

\author{
Scott J. Kenyon \\ Smithsonian Astrophysical Observatory, Mail Stop 15, 60 Garden St. \\ Cambridge MA 02138 \\ Patrick Hartigan \\ Department of Physics and Astronomy, Rice University, 6100 S. Main, \\ Houston TX 77005-1892
}

\begin{abstract}
We describe preliminary results of an HST survey of premain sequence binary stars in the Taurus-Auriga cloud. We use STIS medium and low resolution spectra to derive the reddening, spectral type, and veiling for each component of close pairs with angular separations $\lesssim$ $1^{\prime \prime}$. This sample yields a frequency of mixed pairs, containing one classical and one weak emission $\mathrm{T}$ Tauri star, similar to that of wide pre-main sequence binaries. The components of most pairs are coeval, but the secondaries in four pairs are significantly younger than the primaries for the three sets of evolutionary tracks we considered.
\end{abstract}

\section{Introduction}

Circumstellar disks are common among pre-main-sequence (PMS) stars. PMS disks are strong infrared and millimeter sources (e.g., Osterloh \& Beckwith 1995); CO emission sometimes indicates circular motion around the central star (e.g., Beckwith \& Sargent 1996). Stars with circumstellar disks often show signs of accretion, such as strong $\mathrm{H} \alpha$ emission with inverse P-Cygni profiles (Edwards etal 1994), and ultraviolet and optical veiling emission (e.g., Bertout, Basri \& Bouvier 1988; Hartigan etal 1991). Accretion disks have a profound influence on young stars and their environments in regulating the angular momentum of infalling material (Herbst etal 2000), providing a birthplace for planets (e.g., Kenyon \& Luu 1999), and collimating stellar jets and outflows (e.g., Shu etal 1996). Reipurth \& Bertout (1997) and Lada \& Kylafis (1999) contain reviews of the formation and evolution of disks and jets associated with pre-main sequence stars.

Recent surveys show that most PMS stars are binary systems with separations of $10 \mathrm{AU}-200 \mathrm{AU}$ (Ghez, Neugebauer \& Matthews 1993). Binary motion in these systems may disturb or disrupt a circumstellar disk with a typical radius of $\sim 100$ AU (O'Dell \& Wen 1994). A good example is UZ Tau, a quadruple system consisting of two binaries separated by $\sim 500 \mathrm{AU}$. One pair, UZ Tau E, is a spectroscopic binary (separation 0.03 AU; Mathieu, Martin \& Maguzzu 1996) with a large amount of circumstellar material. The other pair, UZ Tau W, has a 
separation of $50 \mathrm{AU}$ and shows no evidence for disk emission (Jensen, Koerner \& Mathieu 1996). The orbit of the UZ Tau W binary probably leads to the disruption of the disk on a short timescale; the UZ Tau E binary can retain a large disk beyond the very close pair. DQ Tau is another example of a binary interacting with its disk. Accretion appears pulsed in phase with the orbital period of the binary (Basri, Johns-Krull \& Mathieu 1997), perhaps the result of streams of material that flow onto the stars through a central gap evacuated in the disk by the stars (Artymowicz \& Lubow 1996).

We have begun a spectroscopic survey of close binary PMS in Taurus-Auriga using HST. The STIS spectrograph on HST cleanly resolves spectra of close pairs with angular separations of 0 " 1 to $1 . \prime 0$, which is $14 \mathrm{AU}-140 \mathrm{AU}$ at the distance of the Taurus-Auriga dark clouds. STIS spectra allow us to probe the scale where a binary can disrupt a disk and potentially halt accretion during the earliest stages of PMS evolution. The primary goals of this project are to investigate (1) whether close binaries are coeval on PMS evolutionary tracks (as are most of their wider counterparts, Hartigan, Strom \& Strom 1994; Brandner \& Zinnecker 1997), (2) determine whether mixed pairs, where one star has a disk and the other does not, are equally rare among close pairs as among wide pairs (Prato \& Simon 1997), (3) measure whether mass accretion rates are higher onto the secondaries, as some models predict (Artymowicz \& Lubow 1996), and (4) otherwise characterize the accretion and disk activity among the stars in each pair. The program is currently about half complete, with the remaining observations scheduled for the fall and winter of $2000 / 2001$. This paper provides a progress report on the existing data.

\section{Observations}

Our program GO-7310 consists of 22 orbits. We plan observations of 15 pairs of classical T Tauri stars (cTTs) with angular separations of $1^{\prime \prime}$ or less. The remaining observations are of weak emission T Tauri stars (wTTs) to serve as templates for the photospheres of cTTs in our veiling measurements (see §4). Most of the wTTs are also close binaries, which improves our coevality test for PMS binaries. All wTTs pairs have been observed, but only half of the cTTs pairs have complete data. We acquired a low resolution spectrum and a medium resolution spectrum each orbit. The low resolution setting uses the G750L grating and covers $5236 \AA$ to $10266 \AA$; the medium resolution setting uses the G750M grating and covers $5966 \AA$ to $6538 \AA$. In addition to the standard pipeline reduction, we observed a special flat field to remove interference fringes at long wavelengths at low resolution. We also correct for hot pixels not removed by the pipeline, and extract blended spatial profiles in the long slit data in an optimal manner.

\section{Analysis}

To measure the accretion and stellar luminosity, we need reliable estimates for the spectral type, the optical reddening, and the veiling for each star. Without a calibrated MK system for PMS stars, we rely on comparisons with calibrated main sequence (MS) and giant stars to derive spectral types and reddening. 

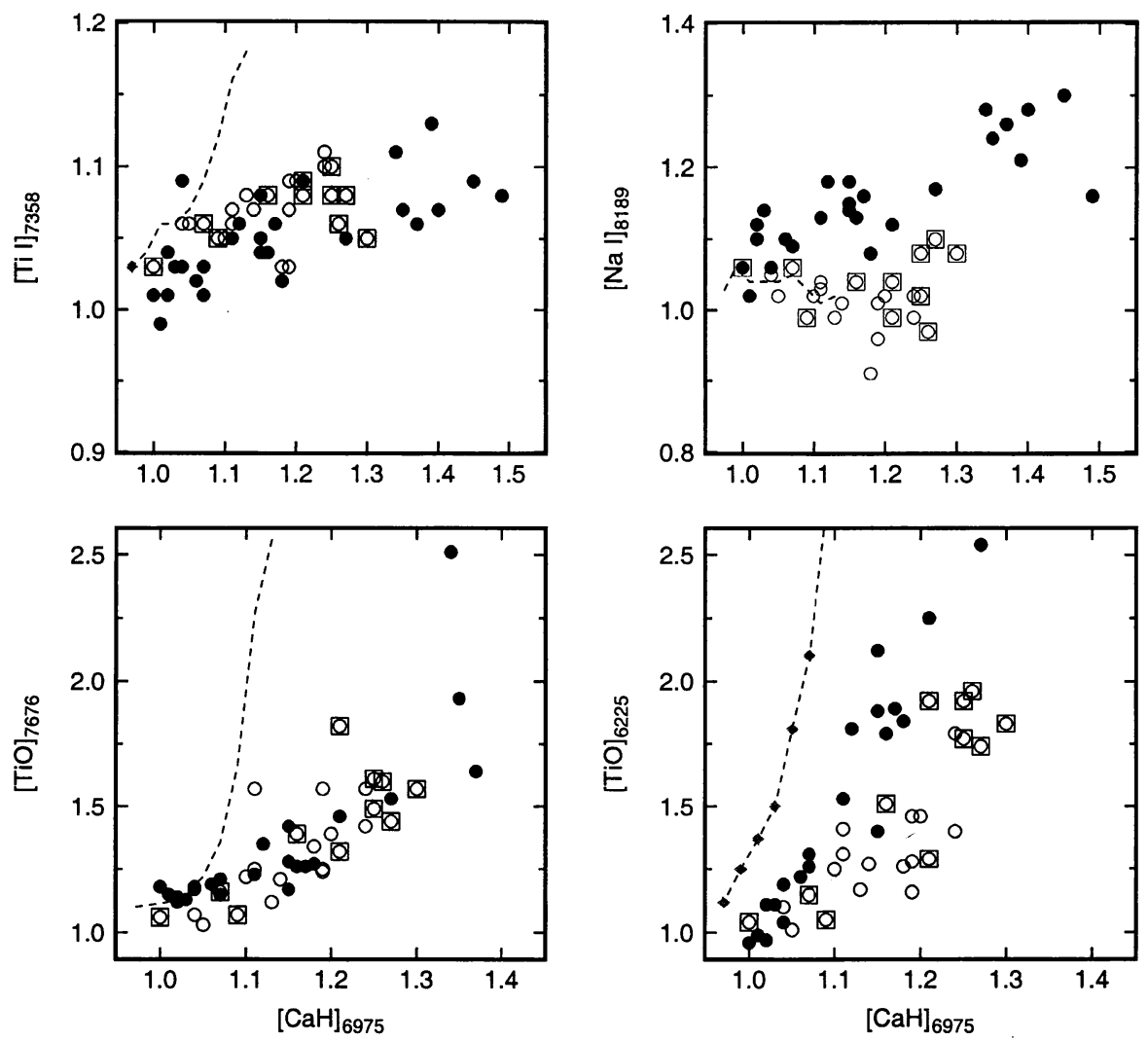

Figure 1. Absorption indices for main sequence stars (filled circles), cTTs (open circles), wTTs (open circles enscribed in open boxes), and red giant stars (dashed lines).

Figure 1 shows four absorption line indices as a function of the $\mathrm{CaH}$ index, defined by Kirkpatrick etal (1991). The $\mathrm{CaH}$ index is a good indicator of spectral type for MS stars. For PMS stars, TiO $\lambda 7676$ and Ti I $\lambda 7358$ follow the MS locus; Na I $\lambda 8169$ follows the giant locus. $\mathrm{TiO} \lambda 6225$ is weaker than bands observed in MS and giant stars. These conclusions are independent of veiling: cTTs and wTTs follow the same locus in each diagram. Although not shown in the Figure, the $m_{5400}-m_{7035}$ color follows the MS locus. We adopt the MS relations between $\mathrm{TiO} \lambda 7676$ and $\mathrm{CaH}$ to derive spectral type and the $m_{5400}-$ $m_{7035}$ color to derive reddening for wTTs. However, we note that other spectral features suggest MS stars are not good matches to the spectra of T Tauri stars.

To measure veiling in a cTTs, we adopt a simple model for the excess flux

$$
F_{v}(\lambda)=a_{0}+a_{1}\left(\lambda-\lambda_{0}\right)+a_{2}^{2}\left(\lambda-\lambda_{0}\right)^{2},
$$

where $a_{0}, a_{1}$, and $a_{2}$ are constants. The predicted spectrum is $F_{p}(\lambda)=c F_{t}(\lambda)+$ $F_{v}(\lambda)$, where $F_{t}$ is the spectrum of a wTT template with the same spectral type as the object. The veiling at $\lambda_{0}$ is $r_{v}\left(\lambda_{0}\right)=F_{v}\left(\lambda_{0}\right) / c F_{t}\left(\lambda_{0}\right)$. We use $\lambda_{0}=6100 \AA ;$ this region has good $\mathrm{S} / \mathrm{N}$ and lies shortward of a strong TiO 

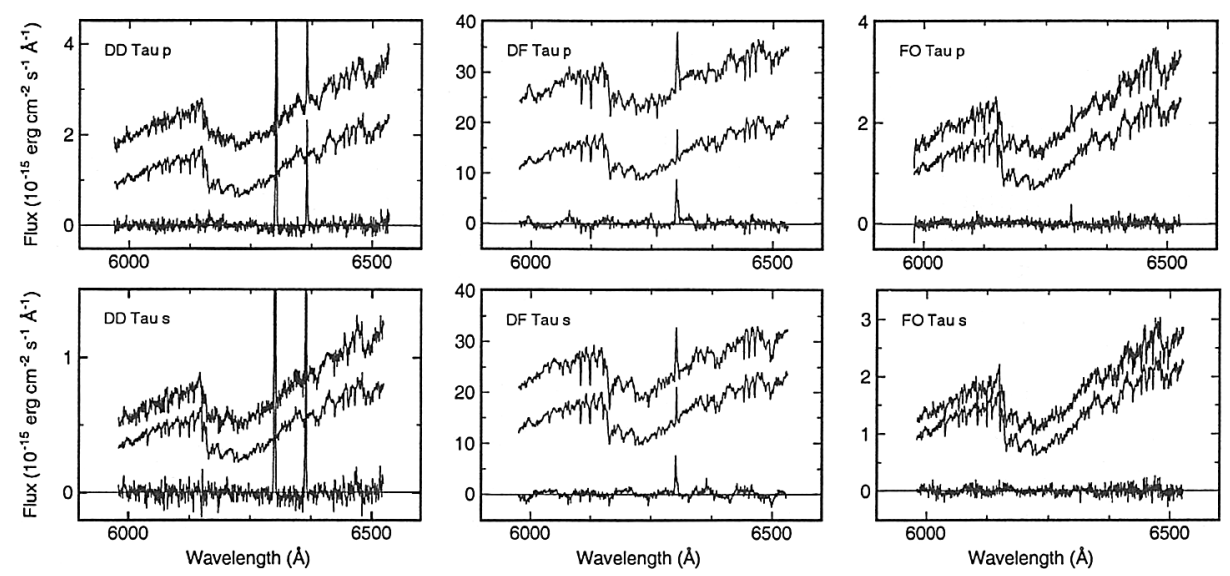

Figure 2. HST spectra for the DD Tau, DF Tau, and FO Tau binaries. The upper panels show results for the primary stars (' $\mathrm{p}$ '); the lower panels show results for the secondary stars ('s').

band. We use an amoeba algorithm (Press etal 1992) to minimize the residuals, $S=\Sigma\left(\left(F_{o}(\lambda)-F_{p}(\lambda)\right) / \sigma(\lambda)\right)^{2}$ where $F_{o}$ is the observed spectrum, as a function of $c, a_{0}, a_{1}$, and $a_{2}$. We set $a_{2} \equiv 0$ for medium resolution data. Tests with $a_{1} \equiv 0$ for medium resolution spectra and $a_{2} \equiv 0$ for low resolution spectra yield similar results.

To estimate stellar parameters, we use colors and bolometric corrections for main sequence stars. We start with the stellar flux at $6100 \AA$ derived from the veiling model, $c F_{t}(6100)$, and compute a reddening-corrected $\mathrm{R}$ magnitude from the $m_{6100}-\mathrm{R}$ relation for main sequence stars. The bolometric magnitude and stellar luminosity follow from the bolometric magnitude-spectral type relation (Kenyon \& Hartmann 1995; KH95) and a distance estimate (d = 140 pc; Kenyon etal 1994). We measure [O I] fluxes from the residual medium resolution spectra and $\mathrm{H} \alpha$ fluxes from the residual low resolution spectra. Reddening-corrected luminosities follow from the reddening, a standard interstellar extinction curve, and the distance.

Figure 2 shows examples of the veiling analysis for three cTTs pairs. In each panel, the upper curve is the observed STIS spectrum. The middle curve is the wTTs template spectrum, scaled to match the absorption line depth in the observed spectrum. The difference between the upper and middle spectrum is the veiling spectrum, which is modeled by equation (1). The lower curve is the residual spectrum derived from the difference between the observed spectrum and the model spectrum. In most cases, the residual spectrum has no obvious features aside from emission lines, such as [O I], visible in cTTs but not in wTTs. In all but 1 or 2 cases, the residual spectrum contains no photospheric features when we use the correct wTTs spectrum for the template. Tests with different templates yield uncertainties in the spectral type ( 0.5 subclasses), veiling $(0.1$ or better), and the reddening ( $0.3 \mathrm{mag}$ or better from low resolution spectra). Tests with wTTs confirm these estimates. 
Table 1 lists results derived from the HST spectra. Hartigan \& Kenyon (this volume) discuss differences between these results and those derived for the combined pair. The reddening difference between components of the same pair is large in LkH $\alpha 332 / \mathrm{G} 2\left(\delta A_{V} \approx 1.4 \mathrm{mag}\right)$, UY Aur $\left(\delta A_{V} \approx 2.0 \mathrm{mag}\right)$, and V927 Tau $\left(\delta A_{V} \approx 0.5 \mathrm{mag}\right)$. Joy \& van Biesbrock (1944) previously noted a large and variable reddening in UY Aur s; our results for $\mathrm{LkH} \alpha 332 / \mathrm{G} 2$ and V927 Tau are new.

Stars with large veiling typically have large $\mathrm{H} \alpha$ and [O I] luminosities. The most heavily veiled, luminous stars - such as the DD Tau and DF Tau pairs - have $\mathrm{H} \alpha$ luminosities of $10^{-3} \mathrm{~L}_{\odot}$ and [O I] luminosities of $10^{-4} \mathrm{~L}_{\odot}$. Stars with smaller amounts of veiling have smaller $\mathrm{H} \alpha$ and [O I] luminosities. The $\mathrm{LkH} \alpha 332 / \mathrm{G} 2$ secondary is an interesting exception: it has a weak $\mathrm{H} \alpha$ line and is modestly veiled. Tests with other templates and other veiling models yield the same results (see below). Two other secondaries - V807 Tau s and V955 Tau s - have modest [O I] emission but are not veiled and lack strong $\mathrm{H} \alpha$ emission.

Table 1. Results from HST Spectra

\begin{tabular}{lcccccc}
\hline \hline TTS & Sp Type & $A_{V}$ & $r_{6100}$ & $\log L_{\star}$ & $\log L_{H \alpha}$ & $\log L_{[O I]}$ \\
\hline DD Tau p & M3.5 & 2.1 & +0.70 & 0.34 & -2.7 & -4.3 \\
DD Tau s & M3.5 & 2.9 & +0.41 & 0.29 & -2.4 & -4.1 \\
DF Tau p & M2.0 & 0.6 & +0.82 & 0.47 & -2.7 & -4.4 \\
DF Tau s & M2.5 & 0.8 & +0.51 & 0.66 & -2.6 & -4.5 \\
FO Tau p & M3.5 & 1.8 & +0.38 & 0.30 & -2.9 & -5.7 \\
FO Tau s & M3.5 & 1.9 & +0.25 & 0.30 & -3.2 & - \\
FQ Tau p & M3.5 & 1.8 & -0.01 & 0.28 & -3.9 & - \\
FQ Tau s & M3.0 & 2.0 & +0.58 & 0.20 & -3.2 & -5.6 \\
FV Tau/c p & M2.5 & 3.3 & -0.09 & 0.18 & -3.7 & - \\
H6-28 p & M2.0 & 2.3 & +0.60 & 0.09 & -3.3 & - \\
H6-28 s & M3.5 & 1.9 & +0.15 & 0.03 & -3.8 & -5.9 \\
HBC 358 p & M3.5 & 0.1 & +0.06 & 0.12 & -4.3 & - \\
HBC 358 s & M4.0 & 0.1 & -0.06 & 0.12 & -4.2 & - \\
HBC 360 p & M3.5 & 0.1 & +0.03 & 0.12 & -4.0 & - \\
LkCa 7 p & K7 & 0.3 & +0.04 & 0.35 & -3.7 & - \\
LkCa 7 s & M3.5 & 0.4 & -0.03 & 0.18 & -4.1 & - \\
LkH $2332 / G 2 ~ p ~$ & M0.5 & 2.0 & - & 0.24 & -4.4 & - \\
LkH $2332 / G 2 ~ s ~$ & M2.5 & 3.4 & +0.20 & 0.15 & -4.3 & - \\
UY Aur p & K7 & 0.7 & +0.26 & 0.27 & -2.9 & -4.4 \\
UY Aur s & M2.5 & 2.7 & +0.54 & 0.17 & -3.1 & -4.7 \\
V807 Tau p & K5 & 0.5 & -0.05 & 2.30 & -2.7 & - \\
V807 Tau s & M2.0 & 0.6 & -0.02 & 1.04 & -3.6 & -4.1 \\
V927 Tau p & M2.0 & 1.4 & +0.02 & 0.32 & -4.0 & - \\
V927 Tau s & M3.0 & 0.9 & +0.07 & 0.11 & -4.9 & - \\
V955 Tau p & K7 & 1.5 & -0.10 & 0.20 & -3.8 & - \\
V955 Tau s & M2.5 & 1.4 & +0.00 & 0.09 & -4.5 & -5.6 \\
\hline \hline
\end{tabular}




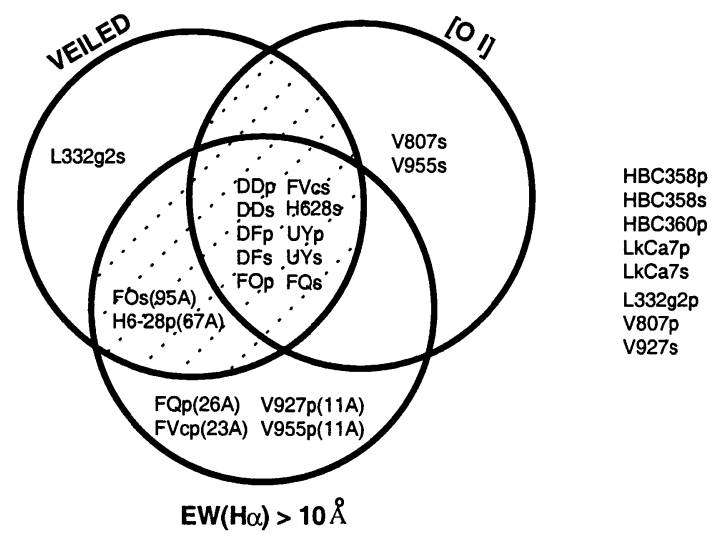

Figure 3. A Venn diagram of the component stars in our sample, sorted according to three disk accretion indicators - optical excess veiling emission $(>0.1)$, detectable [O I] $\lambda 6300$ emission, and large

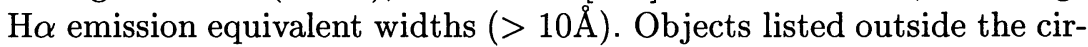
cles have none of the three disk accretion indicators, and are weak-lined $\mathrm{T}$ Tauri stars. We define objects within the dashed area to be cTTs. The $\mathrm{H} \alpha$ equivalent widths for cTTs that lack either veiling or [O I] are given in parentheses.

\section{Frequency of Mixed Pairs}

The so-called 'mixed pairs', a binary with one cTTs and one wTTs, account for only $\sim 15 \%$ of wide binaries (Prato \& Simon 1997; Duchêne etal 1999). Our spectra have three distinct diagnostics of circumstellar disks, continuum veiling, $\mathrm{H} \alpha$ emission, and [O I] emission. Continuum veiling is an excellent indicator of a cTTs if the excess can be measured reliably and if the photospheric template matches the observed lines well. We can measure veiling to an accuracy of 0.1 or better, except in one or two pathological cases. Prominent $\mathrm{H} \alpha$ emission has long been recognized as a cTT indicator. although enhanced chromospheric activity in late-type stars can sometimes exceed the $10 \AA$ threshold usually taken as a discriminant between cTTs and wTTs (Martin 1998). Finally, [O I] $\lambda 6300$ emission has been linked to disks and outflows (Hartigan, Edwards \& Ghandour 1995).

To classify PMS stars, we would like to have all three indicators present (cTTs) or absent (wTTs). Fig. 3 shows that $2 / 3$ of the stars in our sample either have all three disk diagnostics or have none of them. Of the remaining stars, two are veiled and have strong $\mathrm{H} \alpha$, but lack [O I]; , we classify these as cTTs. Four stars have $\mathrm{EW}(\mathrm{H} \alpha)>10 \AA$ but lack $[\mathrm{O} \mathrm{I}]$ and veiling. We classify these as wTTs. Two of these stars have $\mathrm{EW}(\mathrm{H} \alpha)=11 \AA \mathrm{EW}$; the other two (FV Tau/c p, M2.5; FQ Tau p, M3.5) have late spectral types. The $\mathrm{H} \alpha$ in these stars probably arises from chromospheric activity. The only significantly anomalous object is the $\mathrm{Lk} \mathrm{H} \alpha 332 / \mathrm{G} 2$ secondary, which has a veiling of $0.2 \pm$ 0.05 , but lacks $\mathrm{H} \alpha$ and [O I] emission. Our veiling model for this star leaves weak 


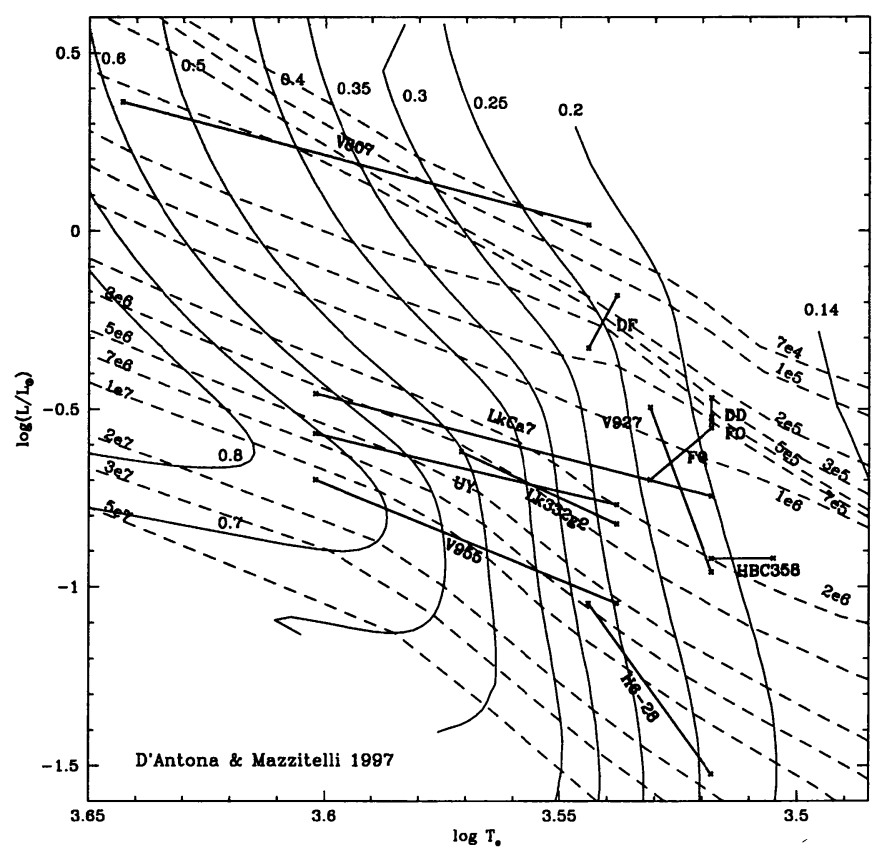

Figure 4. Comparison of HR diagram for PMS binaries with predictions from the D'Antona \& Mazzitelli (1997) tracks.

absorption features in the residual spectrum; light from an unresolved companion or a photospheric anomaly might cause the measured veiling. Without another accretion signature, we classify $\mathrm{LkH} \alpha 332 / \mathrm{G} 2$ as a wTTs.

The current sample yields a mixed pair frequency similar to that of wider PMS binaries. Our classification results in 6 wTTs pairs, 5 cTTs pairs, and two mixed pairs. In both mixed pairs, FV Tau/c and FQ Tau, the primary is a wTTs and the secondary is a cTTs. The $\mathrm{H} \alpha$ luminosity is also larger for the secondary than for the primary in two out of the five cTTs pairs. We will revisit these issues once the entire sample has been observed.

\section{Ages of Binary Components}

Fig. 4-6 compares positions of the PMS binaries with the predictions of three popular sets of theoretical tracks. The primaries and secondaries of several pairs, including DD Tau, FO Tau, and even DF Tau, are coeval using any set of tracks, because their separations in the HR diagram are small compared to the uncertainties, typically \pm 0.2 in $\log \mathrm{L}$ and 0.01 in $\log \mathrm{T}$. The Haro 6-28 and Lk H $\alpha 332 \mathrm{~g} 2$ pairs are nearly coeval for all three tracks; V 927 Tau appears to have an older secondary, but the two stars have identical ages given the observational errors.

Four pairs, V807 Tau, LkCa7, UY Aur, and V955 Tau have secondaries which are significantly younger than the primary on all tracks. In at least one 


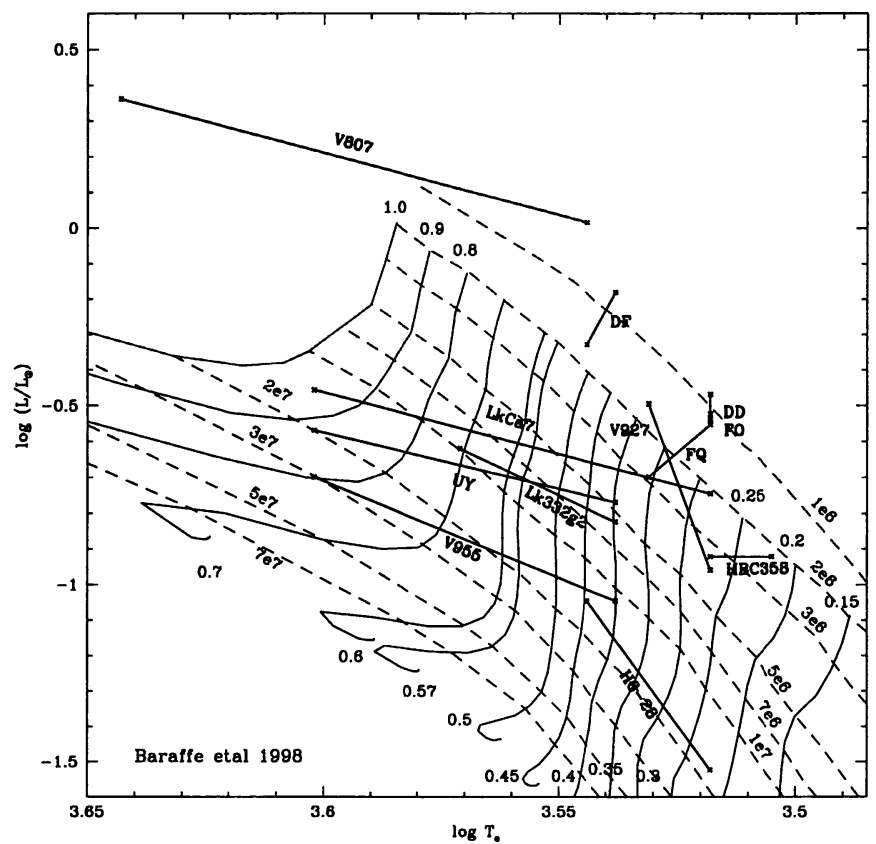

Figure 5. Comparison of HR diagram for PMS binaries with predictions from the Baraffe etal. (1998) tracks.

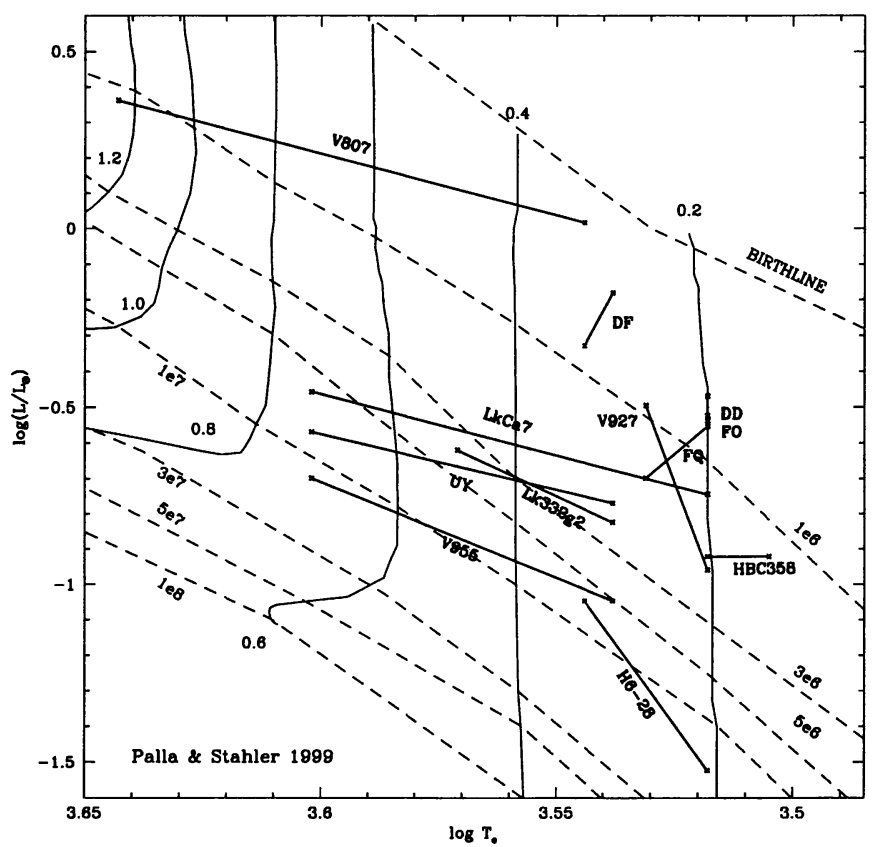

Figure 6. Comparison of HR diagram for PMS binaries with predictions from the Palla \& Stahler (1999) tracks. 
case (V807 Tau), the secondary is itself a very close binary, which accounts for the age difference (Hartigan \& Kenyon, this volume). It is not known whether or not the secondaries of LkCa7, UY Aur, and V955 Tau are also binaries.

\section{References}

Artymowicz, P., \& Lubow, S. 1996, ApJ, 467, L77

Baraffe, I., Chabrier, G., Allard, F., \& Hauschildt, P. H. 1998, A\&A, 337, 403

Basri, G., Johns-Krull, C., \& Mathieu, R. 1997, AJ, 114, 781

Beckwith, S. V. W., \& Sargent, A. I. 1996, Nature, 383, 139

Bertout, C., Basri, G., \& Bouvier, J. 1988, ApJ, 330, 350

Brandner, W., \& Zinnecker, H. 1997, A\&A, 321, 220

D'Antona, F., \& Mazzitelli, I. 1997, Evolution of Low Mass Stars in Cool Stars in Clusters and Associations, ed. R. Pallavicini \& G. Micela, Mem. S. A. It., 68,807

Duchêne, G., Monin, J.-L., Bouvier, J., \& Menard, F. 1999, A\&A, 351, 954

Edwards, S., Hartigan, P., Ghandour, L., \& Andrulis, C. 1994, AJ, 108, 1056

Ghez, A., Neugebauer, G., \& Matthews, K. 1993, AJ, 106, 2005

Hartigan, P., Edwards, S., \& Ghandour, L. 1995, ApJ, 452, 736

Hartigan, P., Kenyon, S., Hartmann, L., Strom, S., Edwards, S., Welty, A., \& Stauffer, J. 1991, ApJ, 382, 617

Hartigan, P., Strom, K. M., \& Strom, S. E. 1994, ApJ, 427, 961

Herbst, W., Rhode, K., Hillenbrand, L., \& Curran, G. 2000, AJ, 119, 261

Jensen, E., Koerner, D., \& Mathieu, R. 1996, AJ, 111, 2431

Joy, A. H., \& van Biesbrock, G. 1944, PASP, 56, 119

Kenyon, S. J., Dobrzycka, M., \& Hartmann, L. 1994, AJ, 108, 1872

Kenyon, S. J., \& Hartmann, L. W. 1995, ApJS, 101, 177 (KH)

Kenyon, S. J., \& Luu, J. X. 1999, AJ, 118, 1101

Kirkpatrick, J. D., Henry, T. J., \& McCarthy, Jr, D. W. 1991, ApJS, 77, 417

Lada, C. J., \& Kylafis, N. D. 1999, The Origin of Stars and Planetary Systems, Proceedings of a NATO Advanced Study Institute, Dordrecht, Kluwer

Martin, E. 1998, AJ, 115, 351

Mathieu, R., Martin, E., \& Maguzzu, A. 1996, BAAS, 188, 6005

O'Dell, C. R., \& Wen, Z. 1994, ApJ, 436, 194

Osterloh, M., \& Beckwith, S. V. W. 1994, ApJ, 439, 288

Palla, F., \& Stahler S. 1999, ApJ, 525, 772

Prato, L., \& Simon, M. 1997, ApJ, 474, 455

Press, W. H., Flannery, B. P., Teukolsky, S. A., \& Vetterling, W. T. 1992, Numerical Recipes, The Art of Scientific Computing, Cambridge, Cambridge

Reipurth, B., \& Bertout, C. 1997, Herbig-Haro Flows and the Birth of Stars, IAU Symposium No. 182, Dordrecht, Kluwer

Shu, F. H., Najita, J., Ostriker, E. C., Shang, H. 1996, ApJ, 45, L155 